\title{
New constraints on reionization from a redshift-independent efficiency model
}

\author{
Rohan Potham Naidu
}

Harvard University, USA

\begin{abstract}
We present an empirical model built on a high-resolution N-body dark matter simulation. We assume a redshift-independent star-formation efficiency for each halo to convert the accretion rate into a star-formation rate. Our model is calibrated using the $z=4$ UV luminosity function (UVLF) and successfully predicts the observed UVLF at $z=5-10$. We present predictions at $z=5-10$ for UV luminosity and stellar mass functions, JWST number counts, the stellar-to-halo mass relation and star-formation histories. We combine this model with bleedingedge reionization constraints (from $z>7$ quasars, $z \sim 7$ Ly $\alpha$ line-profiles, the updated Planck $\tau$ ) to find new perspectives on the Epoch of Reionization (EoR). We find $M_{\mathrm{UV}}<-13.5$ galaxies need an average $f_{\text {esc }}=0.22 \pm 0.05$ to drive reionization and a highly compressed timeline: the IGM neutral fraction is $[0.9,0.5,0.1]$ at $z=[8.4 \pm 0.2,7.0 \pm 0.2,6.3 \pm 0.2]$. Inspired by the newly assembled sample of Lyman Continuum leakers that unanimously displays higher-than-average star-formation surface density (sigma), we fit a model tying $f_{\text {esc }}$ to sigma. Since sigma grows by $>2.5$ dex over $z=0-8$, our model explains the humble values of $f_{\text {esc }}$ at low-z. We find, strikingly, that $<5 \%$ of galaxies with $M_{\mathrm{UV}}<-18$ account for $>80 \%$ of the reionization budget. We predict leakers like COLA1 $\left(z=6.6, M_{\mathrm{UV}}=-21.5\right)$ become common towards the EoR and that the protagonists of reionization are not hiding across the faint-end of the luminosity function but are already known to us.
\end{abstract}

